\title{
REDUCING FLATULENCE FACTORS IN SOYMILK USING COCONUT KERNEL $\alpha$-GALACTOSIDASE IMMOBILIZED HYDROPHOBIC GELS
}

\author{
MADUSHINI NIROSHA DHARMASENA ${ }^{1}$ and C. D. MATHEW ${ }^{*}$ \\ ${ }^{1}$ Department of Chemistry, Faculty of Science, University of Colombo \\ ${ }^{2}$ Department of Biochemistry \& Molecular Biology, Faculty of Medicine, \\ University of Colombo.
}

(Received: 21 June 2001 ; accepted: 25 September 2002)

\begin{abstract}
Alpha- galactosidase from coconut kernel was purified to homogeneity by acidification, ammonium sulfate fractionation and ion exchange chromatography. The purified enzyme had a specific activity of $24.5 \mathrm{units} / \mathrm{mg}$; recovery was $52.6 \%$ and was shown to be homogenous by polyacrylamide gel electrophoresis. The enzyme was immobilized by hydrophobic interactions using four types of ligands bound to sepharose-4B gel. Purified $\alpha$-galactosidase was applied separately to four gels: sepharose-4B-lysine-aminocapronilide, sepharose-4B-urea-alanine-benzoate, sepharose-4B-amino capronilide and sepharose- $4 \mathrm{~B}$-urea caproic. The percentage binding was found to be $1.9 \%, 8.9 \%, 89 \%$ and $85.6 \%$ respectively. The first two gels did not result in significant raffinose hydrolysis capacity whereas the latter two gels hydrolyzed $83.8 \%$ and $48.3 \%$ respectively of a $1 \%$ raffinose solution in four and a half hours at a flow rate of $40 \mathrm{ml} / \mathrm{hour}$. Cellulose thin layer chromatography revealed $2.5 \%( \pm 1.6)$ raffinose, $4.1 \%( \pm 1.3)$ stachyose and $6.6 \%( \pm 2.8)$ total flatulence causing oligosaccharides in soymilk available in the market. When the gels $(10 \mathrm{ml})$ were packed in dialysis tubes and immersed in soymilk $(200 \mathrm{ml})$. with continuous stirring, total flatulence causing oligosaccharides were reduced by $73.3 \%$ and $53.3 \%$ by sepharose-4B-aminocapronilide gel and sepharose-4B-urea-caproic gel respectively.
\end{abstract}

Key Words: Cocos nucifer $\alpha$, flatulence, $\alpha$-galactosidase, immobilized, soymilk

\section{INTRODUCTION}

Soymilk is important as an economical high protein beverage that can help overcome widespread protein deficiencies in developing countries. ${ }^{1}$ Its potential as a substitute for cow's milk has been emphasized over the years, especially for infants and children who are allergic to cow's milk or for adults who have a low level of lactase in their intestinal epithelium. Moreover, soymilk can be used as an economical protein containing beverage when cow's milk is not available or is expensive. On account of its low content of fiber and high nutritional value, soymilk is comparable to cow's milk. $^{2}$ In addition, recent studies have suggested that soymilk may have antineoplastic properties. Thus, soymilk consumption may be protective against hormone dependent cancers, possibly in part due to the isoflavones, daidzein and genistein which are weakly estrogenic. . $^{3,4}$

However, soymilk is deficient in sulphur containing amino acids and contains considerable amounts of the raffinose family oligosaccharides which have been 
implicated as factors responsible for flatulence. Flatulence is a common complaint even among healthy individuals and is one of the common causes of abdominal discomfort. It is also associated with dyspepsia, constipation and diarrhoea. It has been suggested that flatulence is partly caused by microbial fermentation of galactosyl oligosaccharides such as raffinose and stachyose, producing gas and acids. ${ }^{2}$

The raffinose family oligosaccharides cannot be digested by humans due to the lack of $\alpha$-galactosidase in their digestive tract. As a result, virtually all ingested stachyose and raffinose reach the colon. Colonic bacteria possess the enzyme systems necessary to ferment these sugars, and large amounts of carbon dioxide and hydrogen can be adsorbed and excreted in the breath, passed through the rectum, or utilised by other bacterial species for the reduction of carbon dioxide to methane or reduction of sulphates to sulphides. This gas and acid production may play a role in the acceptability of soymilk as a major food source. ${ }^{5}$

Various approaches have been suggested in order to lessen the flatulence factors of soymilk such as development of transgenic soybean plants with low raffinose family oligosaccharides, ${ }^{5}$ removal of these oligosaccharides from soymilk using solvents, ${ }^{6}$ hydrolysis of raffinose family oligosaccharides by immobilized $\alpha$-galactosidase, ${ }^{7}$ microbial fermentation by $\alpha$-galactosidase secreting microorganisms, ${ }^{2}$ ultrafilteration of the aqueous fraction of soymilk etc. ${ }^{5}$ If the gels containing enzyme are packed in glass columns and soymilk is passed through, it cannot be used as a continuous reactor as it can easily get clogged. Thus, gels were packed in dialysis tubes and immersed in soymilk with continuous stirring. The small sugar molecules can diffuse through the dialysis membrane and can get hydrolyzed by the immobilized enzyme.

The enzyme $\alpha$-galactosidase ( $\alpha$-D-galactoside galactohydrolase, E.C. 3.2.1.22) hydrolyses terminal, non-reducing $\alpha$-D-galactose residues in the $\alpha$-galactosides, as well as galactose oligosaccharides. ${ }^{8}$ It hydrolyses the $\alpha-1,6$ linkages in raffinose and stachyose to give sucrose and galactose, thereby reducing flatulence causing components in soymilk.

\section{METHODS AND MATERIALS}

Preliminary purification of $\alpha$-galactosidase: Alpha-galactosidase from coconut kernel was purified according to the method described by Balasubramanium and Mathew. ${ }^{9}$ The enzyme was purified to homogeneity by acidification, ammonium sulfate fractionation and ion exchange chromatography. $\alpha$-galactosidase activity was assayed according to the method described by Dey \& Pridham. ${ }^{10}$ Homogeneity was determined by polyacrylamide gel electrophoresis. ${ }^{9}$

Preparation of hydrophobic gels: Four hydrophobic gels, with a volume of $10 \mathrm{ml}$ each, were prepared. Activation of sepharose- $4 \mathrm{~B}$ gel was carried out according to 
the method described by Cuatrecasas. ${ }^{11}$ Four types of ligands were synthesized (Figure 1). Ligand bindirig was carried out according to the method described by Balasubramanium and Mathew. ${ }^{12}$ The first ligands ( $2 \mathrm{~m}$ Moles) were suspended in $0.2 \mathrm{M} \mathrm{NaHCO}_{3}$ buffer ( $\mathrm{pH} 8.5$ ), and were immediately added to the activated gei suspension, mixed and kept overnight in the cold room $\left(6^{\circ} \mathrm{C}\right)$. Before adding the second ligand, the ligand bound gels were washed with distilled water and the $\mathrm{pH}$ adjusted to 4.5 using $\mathrm{HCl}$. The second ligands ( $2 \mathrm{~m}$ Moles) were dissolved in distilled water $(10 \mathrm{ml})$ or $40 \%$ dimethyl formamide $(10 \mathrm{ml})$ and the $\mathrm{pH}$ was adjusted to 4.5 using $\mathrm{HCl}$. This was added to the gel suspension. All the other ligands were dissolved in distilled water except for aniline, benzoic acid and caproic acid which were dissolved in $40 \%$ dimethyl formamide (10ml). 1-ethyl-3-(3-dimethyl aminopropyl) carbodiimide ( $5 \mathrm{mMoles}$ ) was dissolved in distilled water $(10 \mathrm{ml})$ and the $\mathrm{pH}$ was adjusted to 4.5 using $\mathrm{HCl}$. This was added to the gel suspension. The $\mathrm{pH}$ was maintained around 4.5-5.0. The gel suspension was kept overnight in the cold room.
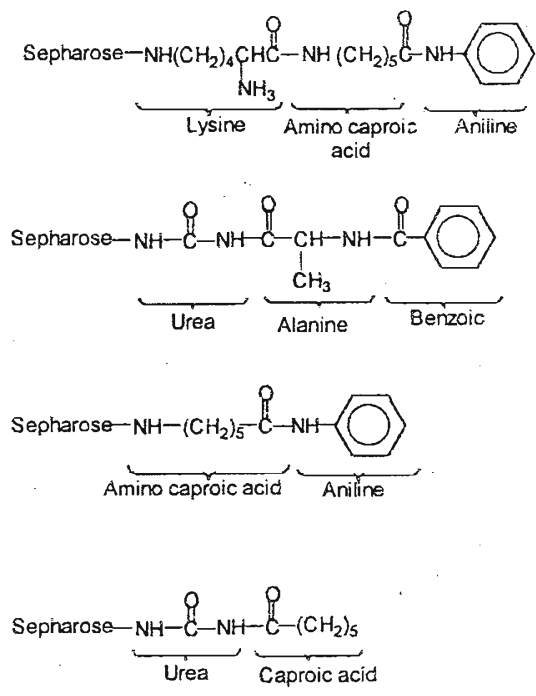

Figure 1: Hydrophobic ligands prepared for immobilization of $\alpha$ galactosidase

Immobilization of $\alpha$-galactosidase: Each gel was equilibrated with $0.01 \mathrm{M}$ Mcllvaine buffer ${ }^{13}$ containing $40 \%\left(\mathrm{NH}_{4}\right)_{2} \mathrm{SO}_{4}(100 \mathrm{ml})$. Purified $\alpha$-galactosidase $(4 \mathrm{ml})$ with the same buffer containing $\left(\mathrm{NH}_{4}\right)_{2} \mathrm{SO}_{4}(6 \mathrm{ml})$ was agitated on a mechanical shaker for 20 minutes. Then the gels were packed into columns and washed with $0.01 \mathrm{M}$ Mcllvaine buffer $(100 \mathrm{ml})$. Fractions $(5 \mathrm{ml})$ were collected and assayed for $\alpha$-galactosidase activity.

Determination of raffinose hydrolysis: The degree of raffinose hydrolysed was determined by assessing the amount of galactose produced. $20 \mathrm{ml}$ of $1 \%$ raffinose 
solution was passed through the $\alpha$-galactosidase bound hydrophobic gels in columns $(1 \times 5 \mathrm{~cm})$ by circulation with a flow rate of $40 \mathrm{ml} /$ hour, and $1 \mathrm{ml}$ of the samples was collected and the galactose content was quantitatively determined according to the method described bv Somogi. ${ }^{14}$

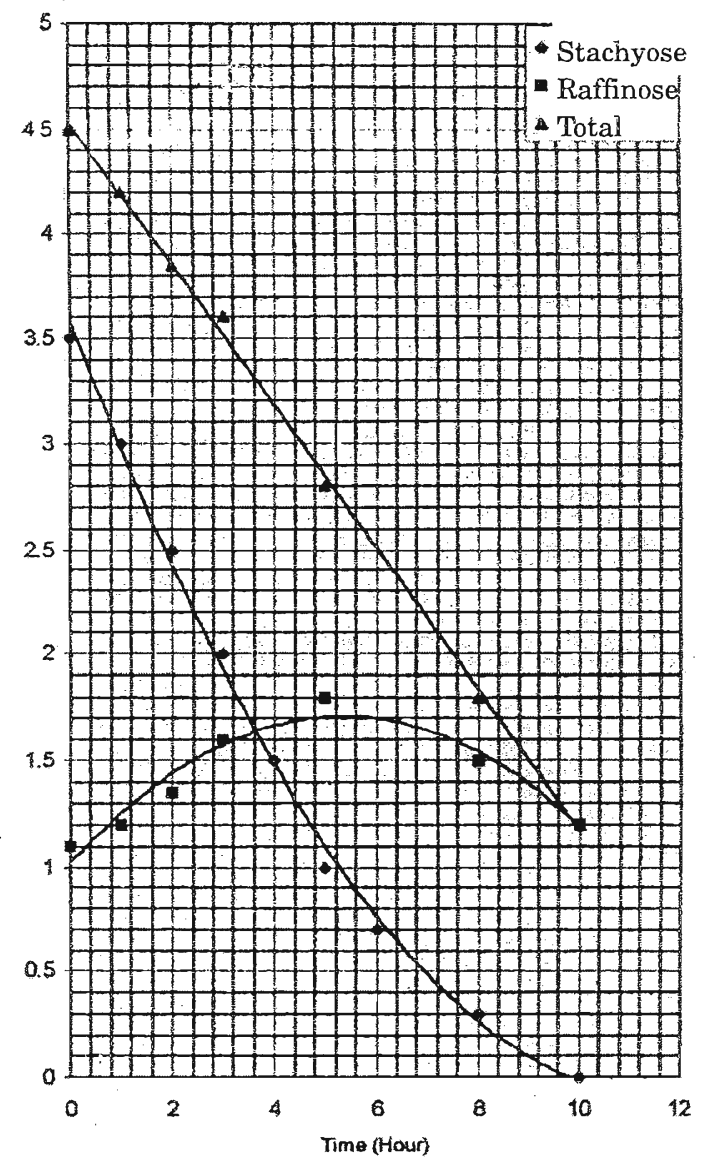

Figure 2: Change in stachyose, raffinose and total flatulence causing oligosaccharide content in soymilk when subjected to hydrolysis by sepharose-4B-caprinilide gel.

Hydrolysis of raffinose in soymilk: Each of the $\alpha$-galactosidase immobilized sepharose gels contained in a dialysis tube was immersed in commercial soymilk (200 ml) with continuous stirring using a magnetic stirrer. Soymilk $(1 \mathrm{ml})$ was removed on an hourly basis upto 10 hours and raffinose and stachyose content determined according to the method described by Tanaka et al. ${ }^{15} 20 \mu \mathrm{l}$ of the sample were applied on a cellulose chromagram sheet and developed by ascending chromatography using a mixture of $n$-propanol, ethylacetate and water $(6: 1: 3 \mathrm{v} / \mathrm{v}) . \alpha$-napthol reagent (1\% $\alpha$-napthol in ethanol; $5 \mathrm{ml}$ of $\mathrm{H}_{3} \mathrm{PO}_{4}$ was added to $50 \mathrm{ml}$ spraying reagent before use) 
was sprayed to locate fructose containing sugars. The guide-strip technique was used to determine the amounts of raffinose and stachyose. The sugar spot was scraped off and the sugar was extracted with $2 \mathrm{ml}$ distilled water in a test tube overnight. Eluent $(1 \mathrm{ml}$ ) containing sugar was mixed with $1 \mathrm{ml}$ of $0.02 \mathrm{M}$ thiobarbituric acid and $1 \mathrm{ml}$ of concentrated HCI. The mixture was heated in a boiling water bath for exactly 6 minutes and cooled under running water. The absorbance of the yellow colour produced was read at $432.5 \mathrm{~mm}$. The experiments were done in triplicate and the concentration of sugar was calculated from working standards, ranging from $100 \mu \mathrm{g}-800 \mu \mathrm{g}$ sugar per $20 \mu \mathrm{l}$.

Raffinose and stachyose content in commercial soymilk: The raffinose and stachyose contents of soymilk from six commercial retail centers were determined by the method described above.

\section{RESULTS}

\section{Purification of $\alpha$-galactosidase}

The purified enzyme had a specific activity of $24.5 \mathrm{units} / \mathrm{mg}$ and the recovery was $52.6 \%$. The purified enzyme was shown to be homogenous by polyacrylamide gel electrophoresis.

\section{Percentage binding and raffinose hydrolysis by hydrophobic gels}

When purified $\alpha$-galactosidase was applied to each of the four gels, sepharose- $4 \mathrm{~B}$ lysine-aminocapronilide, sepharose-4B-urea-alanin-benzoate, sepharose-4Baminocapronilide and sepharose-4B-urea-caproic, the percentage binding was $1.9 \%, 8.9 \%, 89 \%$ and $85.6 \%$ respectively. The first two gels did not have significant raffinose hydrolysis capacity, whereas the latter two gels hydrolyzed $83.8 \%$ and $48.3 \%$ respectively of a $1 \%$ raffinose solution in four and a half hours at a flow rate of $40 \mathrm{ml} /$ hour.

\section{Raffinose family sugar contents in commercial soymilk}

The regression value $(r)$ obtained for the standard curves for raffinose and stachyose are 0.99 and 0.98 respectively. Raffinose and stachyose content in commercial soymilk varies between $1.2 \%-5.4 \%$ and $2.7 \%-6.8 \%$ respectively. Mean values obtained were raffinose $2.5 \%( \pm 1.6)$, stachyose $4.1 \%( \pm 1.3)$ and total flatulence causing oligosaccharides $6.6 \%( \pm 2.7)$.

\section{Hydrolysis of stachyose and raffinose in soymilk}

Sepharose-4B-aminocaprinilide gel and sepharose-4B-urea-caproic gel were used to hydrolyze raffinose and stachyose in soymilk. Total hydrolysis of stachyose was 
observed with a $10 \%$ increase in raffinose within 10 hours in sepharose-4B-caprinilide gel (Figure 2). Similarly, $77 \%$ of stachyose was hydrolyzed and a $27 \%$ increase in raffinose was observed within 10 hours in sepharose-4B-urea-caproic gel (Figure 3). In addition, the total flatulence causing oligosaccharides were reduced to $1.2 \%$ and $2.1 \%$ from $4.5 \%$ in sepharose-4B-aminocapronilide gel and sepharose-4B-urea-caproic acid gel respectively within 10 hours (Figures 2,3 ). No easily detectable changes in colour and aroma were detected even after 10 hours of incubation.

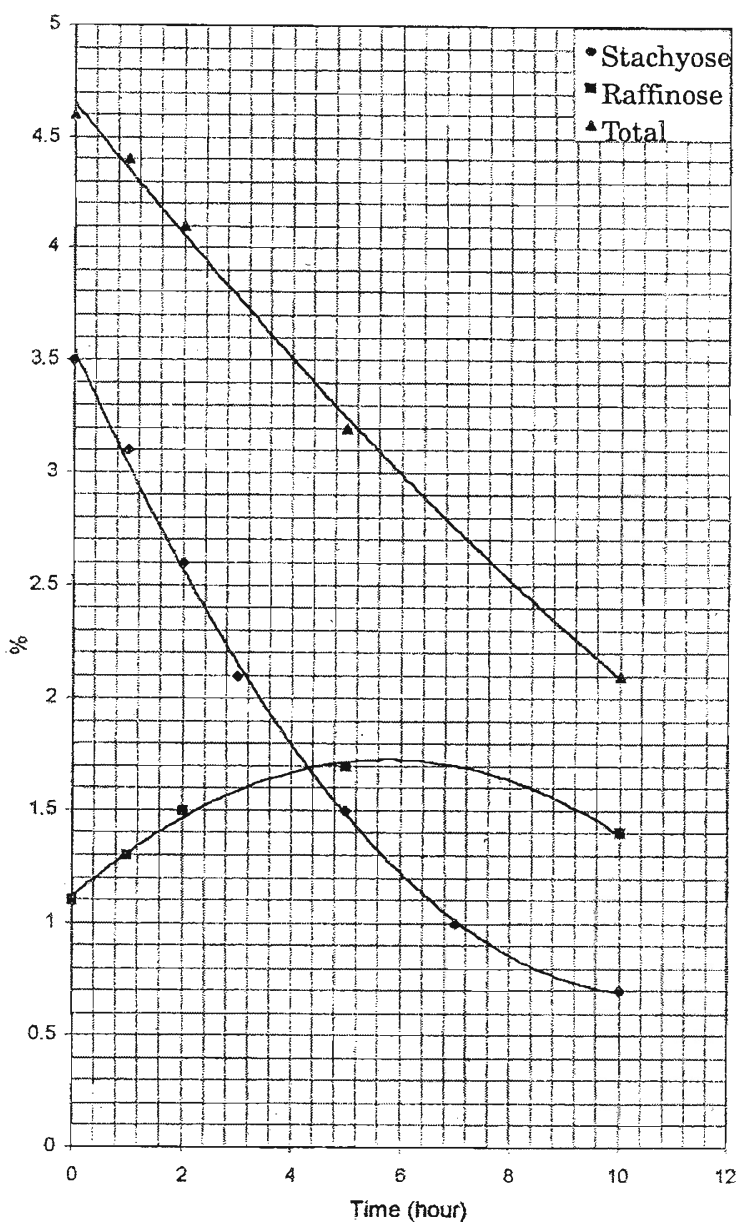

Figure 3: Change in stachyose, raffinose and total flatulence causing oligosaccharide content in soymilk when subjected to hydrolysis by sepharose-4B-urea-caproic gel.

\section{DISCUSSION}

Although $\alpha$-galactosidase was bound to sepharose-4B-aminocapronilide gel and sepharose-4B-urea-caproic gel, the efficiency of raffinose hydrolysis was low. But 
the advantage of the use of hydrophobic gels over other gels in industry is its cost effectiveness due to reusability. Alpha-galactosidase bound to hydrophobic gel can easily be removed when the enzyme is inactivated and replaced with active $\alpha$-galactosidase. Thus, it is not necessary to synthesize ligand bound gel each time the lifetime of the enzyme is over. This would result in considerable saving in terms of cost and time. Further, it gives the advantage of not using CNBr to activate the sepharose- $4 \mathrm{~B}$ gel after the initial activation. Thus, the use of hydrophobic gels in industry to reduce the flatulence causing oligosaccharides is feasible, if the efficiency of the hydrophobic gel can be improved by trying out various ligands.

Exact values cannot be given for raffinose and stachyose content in soymilk, but it can be said that the raffinose and stachyose content in soymilk varies between $1.2 \%-5.4 \%$ and $2.7 \%-6.8 \%$ respectively. Furthermore, in previous research, various scientists had obtained different values for raffinose and stachyose content in soymilk using the same cellulose TLC method used in this study. In 1995, Mulimani and Ramalingam ${ }^{16}$ had obtained $1.95 \%$ for raffinose and $6.1 \%$ for stachyose and Sugimoto and Van Buren ${ }^{1}$ had obtained $1.0 \%$ for raffinose and $3.2 \%$ for stachyose. Different raffinose and stachyose values obtained for different soymilk preparations from various sources may be due to the method of soymilk preparation, thickness or concentration, duration of soaking and the quality of dry beans used. ${ }^{17}$ It is said that the level of oligosaccharides in legume seeds is different among various varieties and strains and rises during maturation of the seeds. ${ }^{2}$ Further, it has been reported that soaking the dry beans in water before preparing soymilk reduces these oligosaccharides ${ }^{10}$, and germination of the seeds results in the utilisation of the bulk of these sugars. ${ }^{5}$

The total raffinose and stachyose content in soymilk was reduced from $4.5 \%$ to $1.2 \%$ by sepharose-4B-aminocaprinilide gel and from $4.5 \%$ to $2.1 \%$ by sepharose-4B-urea-caproic gel. In both gels, a substantial increase in raffinose was observed due to hydrolysis of stachyose in soymilk. However, the decrease in stachyose is more important than the increase in raffinose because the stachyose content in soymilk is 2-5 times higher and also because stachyose induces a higher increase in flatus than raffinose. ${ }^{2}$ If $100 \mathrm{ml}$ each of the (i) unprocessed soymilk, (ii) soymilk processed by sepharose-4B-caprinilide gel or (iii) sepharose-4B-ureacaproic gel is consumed, each portion contains $4.5 \mathrm{~g}, 1.2 \mathrm{~g}$ and $2.1 \mathrm{~g}$ of flatulence causing oligosaccharides respectively.

Suarez et $a l .{ }^{5}$ reported that ingestion of soy products containing $1.32 \mathrm{~g}$ to $3.06 \mathrm{~g}$ of raffinose family oligosaccharide did not significantly increase flatulence frequency. However, ingestion of soy products containing $3.1 \mathrm{~g}$ of raffinose family oligosaccharide resulted in a significant increase in flatulence frequency. As the gas production increases in the colon, a small fraction of the gas is absorbed and the major fraction is excreted through the rectum. Thus, it is possible that a slightly higher dose in raffinose family oligosaccharide might have resulted in a disproportionate increase 
in total gas output and more readily recognizable increase in flatulence frequency. Therefore, there is an apparent threshold value for consumption of raffinose family oligosaccharide. Flatulence can be avoided by keeping the raffinose family oligosaccharide below the threshold value. According to Suarez et al. ${ }^{5}$ gas production in humans ingesting soybean products, consumption of $100 \mathrm{ml}$ of unprocessed soymilk causes a significant increase in flatulence frequency, whereas the processed soymilk may not. Reducing the total raffirose family oligosaccharides to $1-2 \%$ is sufficient to overcome flatulence (assuming around $100 \mathrm{ml}$ of soymilk is consumed at a time). Thus, flatulence is not caused by soymilk processed by sepharose-4Baminocapronilide gel and sepharose-4B-urea-caproic gel.

\section{Acknowledgement}

Department of Chemistry, Faculty of Science and Department of Biochemistry \& Molecular Biology, Faculty of Medicine, University of Colombo, for their support.

\section{References}

1 Sugimoto H. \& Buren J. P. V. (1970). Removal of oligosaccharides from soymilk by an enzyme from Aspergillus saitoi. Journal of Food Science 35: 655-660.

2 Thanankul D., Tanaka D. \& Chichester C. O. (1976). Degradation of raffinose and stachyose in soymilk by $\alpha$-galactosidase from Mortierella vinacea, Entrapment of $\alpha$-galactosidase within polyacrylamide gel. Journal of Food Science 91: 173-174.

3 Lu L. J. \& Anderson K. E.(1998). Sex and long term soy diets affect the metabolism and excretion of soy isoflavones in humans. American Journal of Clinical Nutrition 68(6): 1500-1504.

4 Nagata C., Takatsuka N., Inaba S., Kawakami N. \& Shimizu H. (1998). Effect of soymilk consumption on serum estrogen concentration in premenopausal Japanese women. Journal of National Cancer Institution 90(23): 1830-1835.

5 Suarez F. L., Springfield J., Furne J. K., Lohrmann T. T., Kerr P. S. \& Levitt M. D. (1999). Gas production in humans ingesting a soybean flour derived from beans naturally low in oligosaccharides. American Journal of Clinical Nutrition, 69(1): 135-139.

6 Dey P.M. (1976). Biochemistry of $\alpha$-galactosidic linkages in the plant kingdom. Advanced Carbohydrate Chemistry Journal 37: 283-371. 
7 Mathew C. D. (1985). Immobilization of $\alpha$-galactosidase. Proceedings of SLAAS 41: 86.

8 Dey P. M. \& Pridham J. B. (1972). Biochemistry of $\alpha$-galactosidase. Advanced Enzymology 36: 91-123.

9 Balasubramanium K. \& Mathew C. D., (1982). Purification and study of the kinetic properties of $\alpha$-galactosidase from coconut kernel. Journal of National Science Council of Sri Lanka 10(2): 195-204.

10 Dey P. M. \& Pridham J. B. (1969). Purification and properties of $\alpha$-galactosidase from Vicia faba seeds. Biochemical Journal 113: 49-55.

11 Cuatrecasas P. (1970). Affinity chromatography. Journal of Biological Chemistry 245: 3059-3065.

12 Mathew C. D. \& Balasubramanium K. (1986). Chemical modification of $\alpha$-galactosidase from coconut endosperm. Phytochemistry 26: 1-3.

13 McIlvaine T.C. (1921). A buffer solution for colorimetric comparison. Journal of Biological Chemistry. 49: 183-186.

14 Somogi M. (1945). A new reagent for determination of sugars. Journal of Biological Chemistry 160: 61-68.

15 Tanaka M., Thananunkul D., Lee T. C. \& Chichester C. O. (1975). A simplified method for the quantitative determination of sucrose, raffinose \& stachyose in legume seeds. Journal of Food Science 40: 1087-1088.

16 Mulimani V. H. \& Ramalingam U. (1995). Enzymatic hydrolysis of raffinose and stachyose in soymilk by $\alpha$-galactosidase from Gibberella fujikuroi. Biochemistry \& Molecular Biology International 34: 897-905.

17 Silva H. C. \& Braga G. L. (1982). Effect of soaking and cooking on the oligosaccharide content of dry beans (Phaseolus vulgaris, $L$ ). Journal of Food Science 47: 924-925. 